\title{
Majorana's article on "The value of statistical laws in physics and social sciences"
}

\author{
Rosario Nunzio Mantegna \\ Dipartimento di Fisica e Tecnologie Relative, Università di Palermo \\ Viale delle Scienze, Edificio 18, I-90128, Palermo, Italy \\ E-mail: mantegna@unipa.it
}

\begin{abstract}
This article was found by Majorana’s brother among Ettore Majorana’s files and was published in 1942 after Majorana's disappearance in the international Italian journal Scientia. It is a rather special article in several respects. It was intended to present the point of view of a physicist about the value of statistical laws in physics and social sciences to scholars of a broad spectrum of different disciplines such as sociology and economics. It also considers philosophical aspects related to the nature and value of deterministic and statistical laws in science. In his article, Majorana considers quantum mechanics as an irreducible statistical theory because the theory is not able to describe the time evolution of a single particle or atom in a controlled environment at a deterministic level. He concludes that quantum mechanics suggests that there is an "essential analogy between physics and the social sciences, between which an identity of value and method has turned out”. By analyzing the messages of this paper we conclude that Majorana deserves a great tribute not only for his exceptional achievements in theoretical physics but also for his fresh and original views on the importance and role of statistical laws in physics and in other disciplines such as the social sciences.
\end{abstract}

Ettore Majorana's legacy and the Physics of the XXI century

University of Catania, Italy

5-6 October, 2006 


\section{Introduction}

The centenary of the birth of Ettore Majorana, one of the major Italian physicists of all times, has triggered a series of initiatives. Among them the International Conference on "Ettore Majorana's legacy and the Physics of the XXI century" organized by the Dipartimento di Fisica e Astronomia of University of Catania, Italy was a great occasion to consider Majorana's legacy in Physics and more broadly in sciences and culture. Majorana was not a prolific author. He published 9 articles before his disappearance and a 10th article, whose manuscript was found by Majorana's brother among his files, was published in 1942 after his disappearance in the international Italian journal Scientia, through the interest of his friend Giovanni Gentile Jr. [1]. Nine of these articles concerns research topics of physics of Majorana's time and have been discussed in the other articles of this proceedings volume whereas the 10th article is an article still discussing physics concepts but also considering philosophical and epistemological aspects. Majorana's articles were mostly written in Italian. Only one of them was written in German. Italian is not a widespread language and this limitation has prevented Majorana's work becoming known and correctly evaluated by a vast community of scientists. Last year I have translated the 10th article of Majorana "The value of statistical laws in physics and social sciences" to provide to a broad audience of physicists the possibility of direct access to the article.

The article is a rather special article in several respects. In the original presentation for Scientia, Giovanni Gentile Jr. wrote that the article was originally written for a sociology journal. This article was therefore intended to present the point of view of a physicist about the value of statistical laws in physics and social sciences to scholars of a broad spectrum of different disciplines such as sociology and economics. In his article, Majorana considers quantum mechanics as a fundamental and successful theory able to describe the basic processes involving single particles and atoms. He explicitly considers the theory as a statistical theory because the theory is not able to describe the time evolution of a single particle or atom in a precise environment at a deterministic level. As an example of the lack of determinism in the time evolution of a single system he discusses the case of the decay of a radioactive atom. This lack of determinism at the level of an elementary physical system motivated him to suggest a formal analogy between statistical laws observed in physics and in the social sciences. In his article, he concludes that there is an "essential analogy between physics and the social sciences, between which an identity of value and method has turned out". These words seem to pioneer the view that an investigation of complex systems (indeed this term is literally present in the article) of economic or social origin might be conducted on the same epistemological basis as the modeling of physical systems.

\subsection{Pioneering nature of the article}

His conclusion was considered as rather peculiar and was accepted as a general belief by only a minority of physicists for several years. For example in the book "La vita e l'opera di 
Ettore Majorana” [2], Edoardo Amaldi just wrote a single sentence on this article in a biographical and scientific note of 49 pages. Even today, Majorana's point of view might indeed still be rather unpopular among mainstream physicists, in spite of more than 70 years of quantum mechanics and after some major breakthrough in the fields of critical phenomena and chaos theory.

There is a pioneering nature of this article both from the perspective of physics and economics. From the physics point of view, Majorana took a clear position about the key aspect that quantum mechanics forces scientists to use a statistical description down to events involving single entities. Specifically, he wrote: "Gli aspetti caratteristici della meccanica quantistica, in quanto essa si differenzia dalla meccanica classica sono i seguenti: a) non esistono in natura leggi che esprimano una successione fatale di fenomeni; anche le leggi ultime che riguardano i fenomeni elementari (sistemi atomici) hanno carattere statistico, permettendo di stabilire soltanto la probabilità che una misura eseguita su un sistema preparato in un dato modo dia un certo risultato, e ciò qualunque siano i mezzi di cui disponiamo per determinare con la maggior esattezza possibile lo stato iniziale del sistema. Queste leggi statistiche indicano un reale difetto di determinismo e non hanno nulla di comune con le leggi statistiche classiche nelle quali l'incertezza dei risultati deriva dalla volontaria rinunzia, per ragioni pratiche, a indagare nei più minuti particolari le condizioni iniziali dei sistemi fisici.,"1

From the point of view of economics and social sciences, there is an emphasis on the observation that statistical laws have to be used in economic and social modeling. It should be noted this position was not that of the majority of scholars working in the thirties of the last century in both the disciplines considered. In fact, during the thirties of the last century the interaction between social sciences and natural scientists was developed under the paradigm of celestial mechanics (the only exception to this approach was the one pursued by Louis Bachelier that at that time had no impact on the academy [3]). This interaction goes back to the development of general equilibrium theory pursued by Walras, Pareto, Schlesinger and Wald. The emphasis of Majorana on the intrinsic statistical nature of most of the underlying processes describing natural phenomena suggests that statistical laws should be incorporated into a modeling approach to social phenomena. This approach has eventually found its most famous achievement in finance with the Black and Scholes modeling of option pricing [4].

The topic considered by Majorana in his article is timely today for a series of reasons. First, it should be noted that a cross-disciplinary consensus about the epistemological value of statistical laws in different disciplines is not easily found today. The major paradigm of the

\footnotetext{
1 “The specific aspects of quantum mechanics as compared with classical mechanics are the followings: a) there are no laws in nature which are expressing a fatal succession of phenomena. Basic laws governing elementary phenomena (atomic systems) have a statistical character. They establish only the probability that a measure performed in a prepared system will give a certain result. This occurs in spite of the means of which we dispose to determine the initial state of the system with the highest possible accuracy. These statistical laws indicate a real deficiency of determinism. They have nothing in common with the classical statistical laws where uncertainty of results derive from a voluntary renunciation for practical reasons to investigate the initial conditions of physical systems in the most minute aspects.”
} 
validity of a scientific theory is still based on the falsification procedure of a law. It is undisputable that this approach has been devised having in mind the most characteristic laws in physical sciences, i.e. deterministic laws or laws having a deterministic part (as is the case for quantum mechanics when the time evolution of the wave function is considered or for random walk theory when one considers the statistical description of an ensemble of walks). During the past years it has been progressively realized that such an approach might not be the most appropriate to other disciplines such as, for example, biology. For this important and successful discipline, the nature of the laws (or sometimes theories) is often intrinsically related to the prevalence of indeterminacy owing to the high frequency of stochastic processes unavoidably involved. Moreover a double causality (one related to the external conditions and forces and one governed by the amount of information inherited at the biological level under consideration) is present in most cases [5]. Similar observations are most probably also valid for social sciences.

Physics might certainly benefit from a deeper understanding of the role, necessity and peculiarity of statistical laws in physics. Some of the statistical laws are eventually reinterpreted in terms of more fundamental and deterministic laws. However there are cases when a reduction seems to be impossible. One of these cases is indeed quantum mechanics and other more recent examples concern the topics of chaos and critical phenomena theory. By somewhat inverting the perspective of the relations between physics, biology and the social sciences, it might be worth discussing the possibility that physicists should also start to consider as proper to their discipline the investigation of systems where the basic elements composing the system are in possession of a certain level of internal information and are characterized by a certain ability to react to external stimuli by properly processing the available external information with inherited or adaptive rules. Investigations of this kind have been performed with tools of statistical physics properly adapted or extended. For example, one of these models is the minority game [6] recently investigated within the new research field of econophysics [7].

\subsection{Philosophical reflections}

The article also presents philosophical reflections. A major theme of the article is a critical discussion of determinism in physics. Majorana states "Eppure il determinismo, che non lascia alcun posto alla libertà umana e obbliga a considerare come illusori, nel loro apparente finalismo, tutti i fenomeni della vita, racchiude una reale causa di debolezza: la contraddizione immediata e irrimediabile con i dati più certi della nostra coscienza.” ${ }^{2}$. Majorana do not consider determinism compatible with our personal experience of consciousness. This again might seem a rather peculiar approach but, quite surprisingly, it is not too far from the approach recently followed by philosopher John R. Searle. In his work on "Free will as a problem in neurobiology" [8], Searle states "The only way I know to approach such a problem is to begin by reminding ourselves of what we already know. We know, or at least we think we know, two things that bear on the case. First we know that our experiences of free action contain both

\footnotetext{
2 "The determinism, which does not leave any rule to the human freedom and forces to consider as illusory all the phenomena of life, implicates a real cause of weakness. This is the irremediable and immediate contradiction with the most certain data of our conscience.”
} 
indeterminism and rationality and that consciousness is essential to the forms that these take. Second we know that quantum indeterminism is the only form of indeterminism that is indisputably established as a fact of nature." These premises allows him to conclude that "It is tempting, indeed irresistible, to think that the explanation of the conscious experience of free will must be a manifestation of quantum indeterminism at the level of conscious rational decision making. Previously I never could see the point of introducing quantum mechanics into discussions of consciousness. But here at least is a strict argument requiring the introduction of quantum indeterminism.”. A similar point was proposed by Majorana in his article. In fact he concluded his article by saying "La disintegrazione di un atomo radioattivo può obbligare un contatore automatico a registrarlo con effetto meccanico, reso possibile da adatta amplificazione. Bastano quindi comuni artifici di laboratorio per preparare una catena comunque complessa e vistosa di fenomeni che sia comandata dalla disintegrazione accidentale di un solo atomo radioattivo. Non vi è nulla dal punto di vista strettamente scientifico che impedisca di considerare come plausibile che all'origine di avvenimenti umani possa trovarsi un fatto vitale ugualmente semplice, invisibile e imprevedibile. Se è così, come noi riteniamo, le leggi statistiche delle scienze sociali vedono accresciuto il loro ufficio che non è soltanto quello di stabilire empiricamente la risultante di un gran numero di cause sconosciute, ma sopratutto di dare della realtà una testimonianza immediata e concreta. La cui interpretazione richiede un'arte speciale, non ultimo sussidio dell'arte di governo.”3. Therefore Majorana's motivation to reconsider the value and function of statistical laws in physics and social sciences was motivated by a philosophical reflections about the "irremediable and immediate contradiction (of determinism of physics) with the most certain data of our conscience". This interpretation is corroborated by the biographical note of Edoardo Amaldi referring to this article. In fact, he wrote that after Majorana's travel to Leipzig and Copenhagen concluded during the fall of 1933 "Egli cominciò a frequentare l'Istituto di via Panisperna solo saltuariamente e con il passare dei mesi non venne più affatto: trascorreva sempre più le sue giornate in casa immerso nello studio per un numero di ore del tutto eccezionale. Più che di fisica in quel periodo si interessava di economia politica, di politica, delle flotte dei diversi paesi e dei loro rapporti di forza, delle caratteristiche costruttive delle navi. Al tempo stesso gli interessi filosofici, che sempre erano stati vivi in lui, si erano fortemente accentuati, tanto da spingerlo a meditare a fondo le opere di vari filosofi, in particolare quelle di Schopenhauer. Probabilmente risale a quell'epoca il manoscritto sul valore delle leggi statistiche nella fisica e nelle scienze sociali che, trovato fra le sue carte dal fratello Luciano, fu pubblicato dopo la sua scomparsa a cura di Giovanni Gentile Jr." 4

\footnotetext{
${ }^{3}$ The disintegration of a radioactive atom can force an automatic counter to detect it with a mechanical effect, which is possible thanks to a suitable amplification. Common laboratory set-ups are therefore sufficient to prepare whatever complex chain of rich phenomena, which is controlled from an accidental disintegration of a single radioactive atom. From a scientific point of view nothing prevents to consider that an equally simple, invisible and unpredictable vital fact could be found at the origin of human events. If this is so, as we believe it is, the statistical laws of social sciences increase their function. Their function is not only of empirically establishing the resultant of a great number of unknown causes but above all to provide an immediate and concrete evidence of reality. The interpretation of this evidence requires a special art, which is an important support of the art of government.

4 "He started to go to the Institute of via Panisperna only discontinuously and after some time he did not go to at all. He spent his days at home totally immersed in his study for an exceptional number of hours. During that period rather than about physics he took interest in political economy, politics, naval fleets of several countries, their relative
} 


\subsection{Conclusions}

In summary, the 10th article of Majorana raises the necessity of focusing the attention of several disciplines on the value and nature of statistical laws. From physics, to biology and to social sciences, all the scientific disciplines present statistical laws and scholars of these disciplines need to reflect about their role within each discipline. Majorana noticed that quantum mechanics made clear that a scientific description without statistical laws is impossible. Today there is still a need to assess the status of statistical laws and to consider the validation procedures that are most appropriate to these sorts of laws. Validation procedures probably need to be different from those originally devised having in mind deterministic laws.

The article also contains philosophical reflections. These reflections have some points of contact with recent philosophical thoughts about consciousness and free will.

I hope the occasion of the centenary of the birth of Ettore Majorana will be useful to remember and to reconsider not only his exceptional achievements in theoretical physics but also his fresh and original views on the essential aspects, importance and role of statistical laws in physics and in other disciplines such as the social sciences.

\section{References}

[1] E. Majorana, Il valore delle leggi statistiche nella fisica e nelle scienze sociali, Scientia, Quarta serie, Febbraio-Marzo 1942 pp. 58-66. English translation in E. Majorana, The value of statistical laws in physics and social sciences, Quantitative Finance 5 (2005) 133--140.

[2] E. Amaldi, La vita e l'opera di Ettore Majorana, Accademia Nazionale dei Lincei, Roma 1966.

[3] L. Bachelier, Thèorie de la spèculation, Ph.D. thesis in mathematics, Annales Scientifiques de l'Ecole Normale Supèrieure III-17 (1900) 21--86.

[4] F. Black and M. Scholes, The Pricing of Options and Corporate Liabilities, J. Polit. Econ. 81 (1973) 637--654.

[5] E. Mayr, The autonomy of biology: The position of biology among the sciences, The Quarterly Review of Biology 71 (1996) 97-106.

[6] D. Challet, M. Marsili, Y.-C. Zhang, Minority Games : Interacting Agents in Financial Markets, Oxford University Press, Oxford 2005.

[7] J. D. Farmer, M. Shubik, and E. Smith, Is Economics the Next Physical Science?, Physics Today 58, (2005) 37-42.

[8] J.R. Searle, Free will as a problem in neurobiology, Philosophy 76 (2001) 491-514.

strengths and technical aspects of ships. At the same time, philosophical interests, which were always present to him, started to be more accentuated. He deeply reflected upon the work of various philosophers, in particular about works of Schopenhauer. Probably during this period he wrote the manuscript about the value of statistical laws in physics and social sciences that was found by his brother Luciano among his files and was published after his disappearance through the interest of Giovanni Gentile Jr.”. 